\title{
A detailed characterization of the adult mouse model of glycogen storage disease la
}

\author{
Susan V Salganik', David A Weinstein², Thomas D Shupe', Max Salganik ${ }^{3}$, Dana G Pintilie ${ }^{1}$ and Bryon E Petersen
}

Glycogen storage disease type la (GSDla) is caused by a genetic defect in the hepatic enzyme glucose-6-phosphatase (G6Pase- $\alpha$ ), which manifests as life-threatening hypoglycemia with related metabolic complications. A G6Pase- $\alpha$ knockout (KO) mouse model was generated to study potential therapies for correcting this disorder. Since then, gene therapy studies have produced promising results, showing long-term improvement in liver histology and glycogen metabolism. Under existing protocols, however, untreated KO pups seldom survived weaning. Here, we present a thorough characterization of the G6Pase- $\alpha$ KO mouse, as well as the husbandry protocol for rearing this strain to adulthood. These mice were raised with only palliative care, and characterized from birth through 6 months of age. Once KO mice have survived the very frail weaning period, their size, agility, serum lipids and glycemic control improve dramatically, reaching levels approaching their wild-type littermates. In addition, our data reveal that adult mice lacking G6Pase- $\alpha$ are able to mate and produce viable offspring. However, liver histology and glycogen accumulation do not improve with age. Overall, the reliable production of mature KO mice could provide a critical tool for advancing the GSDla field, as the availability of a robust enzyme-deficient adult offers a new spectrum of treatment avenues that would not be tolerated by the frail pups. Most importantly, our detailed characterization of the adult $\mathrm{KO}$ mouse provides a crucial baseline for accurately gauging the efficacy of experimental therapies in this important model.

Laboratory Investigation (2009) 89, 1032-1042; doi:10.1038/labinvest.2009.64; published online 6 July 2009

KEYWORDS: Glucose-6-phosphatase; GSD1a mouse model; glycogen storage disease; hypoglycemia; metabolic disorder

Glycogen storage disease type Ia (GSDIa) is a metabolic disorder resulting from the mutation of the gene encoding for hepatic glucose-6-phosphatase- $\alpha$ (G6Pase- $\alpha){ }^{1}$ The disorder results from homozygous recessive inheritance, and it is currently estimated to have an incidence of $1: 100000 .^{2}$ The G6Pase enzyme is now well understood as a phosphohydrolase that catalyzes the terminal step in gluconeogenesis and glycogenolysis. ${ }^{3,4}$ The absence of hepatic G6Pase activity impairs endogenous glucose production, and hypoglycemia develops with fasting. As a result, GSDIa is commonly diagnosed within the first few months of life on recurrence of severe hypoglycemia and seizures. ${ }^{5}$ Lactic acidemia is also present during the neonatal period, and severe hepatomegaly often leads to a protruding abdomen. Adolescents with the disease often have short stature, delayed puberty, hyperlipidemia, hepatomegaly and nephromegaly, accompanied by recurrent hypoglycemic attacks. Even with improved treatment, long-term complications remain common., ${ }^{2,6-8}$

A detailed characterization of the molecular origins of GSDIa ${ }^{9}$ allowed for the creation and recognition of GSDIa animal models. ${ }^{10}$ Dr Janice Chou created the G6Paseknockout (KO) mouse in $1993,{ }^{9,10}$ and this strain has since been widely used as a gold standard for studying GSDIa in the laboratory. Although a naturally occurring dog model for G6Pase- $\alpha$ deficiency also exists, ${ }^{11}$ the long lifespan and high level of husbandry required for maintaining these animals has resulted in continued interest in the mouse model.

Gene therapy experiments in both of these animal models have produced clear and promising results in terms of both hepatic glycogen accumulation and histology. ${ }^{1}$ However, the results of these studies were interpreted without regard to untreated control animals of similar age. For this reason, the earlier studies in mice could not evaluate the potential for

\footnotetext{
${ }^{1}$ Department of Pathology, Immunology and Laboratory Medicine and The Program for Stem Cell Biology and Regenerative Medicine, University of Florida College of Medicine, Gainesville, FL, USA; ${ }^{2}$ Division of Pediatric Endocrinology, Department of Pediatrics, University of Florida College of Medicine, Gainesville, FL, USA and ${ }^{3}$ Department of Molecular Genetics and Microbiology, Powell Gene Therapy Center, University of Florida College of Medicine, Gainesville, FL, USA

Correspondence: Bryon E Petersen, PhD, Department of Pathology, Immunology and Laboratory Medicine, University of Florida College of Medicine, BOX 100275,1600 SW Archer Rd, Gainesville, FL 32610-0275, USA.

E-mail: petersen@pathology.ufl.edu
}

Received 18 February 2009; revised 1 June 2009; accepted 2 June 2009 
intrinsic gains in health due to maturation. In response to this gap in our knowledge, we set out to characterize the G6Pase- $\alpha \mathrm{KO}$ mouse as it transitions through the neonatal period to maturity. Here, we show that the afflicted pups are not only capable of surviving the very fragile weaning period, but that they also experience the same age-related physiological improvement often reported by human GSDIa patients. ${ }^{12}$ In particular, mice were consistently observed to improve glycemic control, serum profiles, physical activity and mobility, and showed normal reproductive capacity in both genders. These changes occurred in lieu of any genetic treatment, suggesting that biochemical and behavioral adaptations may contribute to the improvements described in animals that receive gene therapy. We also show that the widely expressed G6Pase- $\beta$ isoform does not undergo upregulation in muscle tissue to compensate for the lack of G6Pase- $\alpha$ in the liver.

\section{MATERIALS AND METHODS \\ Mouse Genotype Determination}

C7/B6 mice bearing a G6Pase- $\alpha$ KO allele (a gift of Dr Janice Chou, NIH, UK) were bred in-house and in accordance with IACUC approved guidelines. All pups were genotyped on the day 3 of life by PCR of DNA from tail snips, using the following primers: wild type, $5^{\prime}$-AAGTCCCTCTGGCCATGC CATGGG- $3^{\prime}$ and $5^{\prime}$-CCAAGCATCCTGTGATAA CTC- $3^{\prime}$; KO, $5^{\prime}$-ATACGCTTGATCCGGCTACCTGCC- $3^{\prime}$ and $5^{\prime}$-CATTTG CACTGCCGGTAG AACTCC-3'. PCR was carried out using the protocol previously outlined by Chou and coworkers. ${ }^{13}$

\section{Phenotypic Characterization}

Mice ranging in age from 0 to 6 months were evaluated continuously with regards to the pathophysiology of G6Pase deficiency. Measured parameters included weight, blood glucose, cholesterol, triglyceride, uric acid (UA) levels, liverand kidney:body weight ratio, glycogen accumulation (liver), G6Pase- $\alpha$ activity (liver) and G6Pase- $\beta$ activity (muscle). Body mass was measured with a pocket scale sensitive to $0.1 \mathrm{~g}$. Blood glucose was checked (tail-bleed), no less than every $8 \mathrm{~h}$, using the Freestyle Flash glucose meter (Abbot Diabetes Care, Inc., Alameda, CA, USA). All animal work was done in accordance with the regulations set by the Institutional Animal Care and Use Committee.

\section{Pre-Weaning Care}

Pups with a glucose reading below $70 \mathrm{mg}$ per $100 \mathrm{ml}$ received $25-150 \mu \mathrm{l}$ of $15 \%$ glucose solution by dorsal subcutaneous injection. The exact volume was determined based on the severity of hypoglycemia. Onward from 14 days of age, pups received ad libitum access to standard mouse chow (2018S Teklad Global 18\% Protein Rodent Diet, Harlan Laboratories, Indianapolis, IN, USA), as well as Nutragel $\AA$ high calorie food supplement (Bio-Serv S4798-TRAY, Bioserv Corporation, San Diego, CA, USA). Afflicted pups that survived the standard weaning period (21 days) were allowed to remain with the mother until day 30 .

\section{Post-Weaning Care}

Both standard chow and Nutragel were available at all times, and glucose was added to the drinking water (5\% final solution) daily. Weights were charted throughout the life of the animal. 100-200 $\mu$ l of $15 \%$ glucose solution was administered subcutaneously if blood glucose was between 70 and $30 \mathrm{mg}$ per $100 \mathrm{ml}$; for lower readings, 200-300 $\mu \mathrm{l}$ glucose was administered IP.

\section{Euthanization and Tissue Collection}

Mice were weighed, and then euthanized by cervical dislocation after sedation with isofluorane. Muscle was immediately excised from the long bones of the hind limbs, followed by removal of the liver and kidney. Collected tissues were partitioned into blocks for fixation/paraffin embedding, fresh-frozen Tissue-Tek OCT (Optimal Cutting Temperature; Andwin Scientific, Addison, IL) embedding and microfuge tubes for immediate snap freezing in liquid nitrogen. Blood samples for analysis of lipid and UA content were collected through heparin-treated syringe and transferred to a Capijet blood collection tube (Terumo Medical Corporation, Somerset, NJ). Samples were placed on ice and submitted to a CLIA approved clinical lab.

\section{Histology}

Paraffin-embedded liver and kidney tissue sections were cut to $6-\mu \mathrm{M}$ thickness, and sections were stained with Hematoxylin \& Eosin, Masson's Trichrome and Periodic Acid Schiff (PAS) reagent for histological evaluation, visualization of fibrosis and glycogen quantitation, respectively. Diastase pretreatment was done on a subset of slides to clear the liver tissue of glycogen and reveal any background staining. Briefly, tissue sections were deparaffinized and hydrated with distilled water, and then submerged for $15 \mathrm{~min}$ in warm diastase solution containing $0.05 \mathrm{~g}$ diastase with $\alpha$-amylase (Ameri. Reagent, Shirley, NY, USA) plus $50 \mathrm{ml}$ distilled water. Slides were rinsed in tap and then distilled water. Aperio image analysis software (Aperio, Vista, CA) was used to quantify the intensity of PAS staining. PAS stain was continued on both diastase treated and non-treated slides concurrently. Statistical significance was determined by ANOVA.

\section{Phosphohydrolase Activity Assay}

The phosphohydrolase activity of both isoforms of G6Pase was measured in the liver and muscle tissue as per the Glucose-6-Phosphatase Activity Assay described by Alfred Harper. ${ }^{14}$ In brief, $150 \mathrm{mg}$ tissue was pulverized in liquid nitrogen and suspended in $6 \mathrm{ml} 0.1 \mathrm{M}$ citrate buffer. For test samples, $100 \mu \mathrm{l}$ G6P-buffer was added to $100 \mu \mathrm{l}$ of homogenate. For control solutions lacking G6P, $100 \mu \mathrm{l}$ tissue homogenate received $100 \mu \mathrm{l}$ of simple citrate buffer. The blank solution contained $100 \mu \mathrm{l}$ citrate buffer plus $100 \mu \mathrm{l}$ G6P solution, without any tissue. Reaction mixtures were incubated for $15 \mathrm{~min}$ at $37^{\circ} \mathrm{C}$, which is the optimal temperature for the $\beta$ isoform, and equally acceptable for Glc-6-Pase- $\alpha .{ }^{15}$ In total, 
$2 \mathrm{ml}$ of $10 \%$ trichloroacetic acid was added to each, and solution was centrifuged for $10 \mathrm{~min}$ at $12000 \mathrm{~g}$. A total of $1 \mathrm{ml}$ supernatant plus $5 \mathrm{ml} 0.002 \mathrm{M}$ molybdate solution were added to fresh tubes for experimental and G6P-free control samples. In total, $1 \mathrm{ml} \mathrm{PO} 4$ standard solution plus $5 \mathrm{ml}$ $0.002 \mathrm{M}$ molybdate were added to fresh tubes for blank solution. Reducing reagent $(1 \mathrm{ml})$ was added to each tube at $1 \mathrm{~min}$ intervals, and the samples were incubated for $15 \mathrm{~min}$ at room temperature. Samples were transferred to 1-ml cuvettes for spectrophotometry at $700 \mathrm{~nm}$, with readouts between 1 and 20 arbitrary units. Each preparation represents an individual mouse, with at least three independent preparations used per assay. Micromoles phosphate liberated is found by the equation, A700 for ((experimental-G6Pase-free control)/ blank $) \times\left(0.5 \mu \mathrm{M} \quad \mathrm{PO}_{4}\right) \times(2.2 \mathrm{ml}$ total $\mathrm{rxn}$ vol $) \times((1000 /$ $(15 \times 2.5))$. Units are $\mu \mathrm{M} \mathrm{PO} 4 / \mathrm{min}$ per gram tissue.

\section{Quantitative Real-Time rt-PCR Analysis of G6Pase- $\beta$}

G6Pase- $\beta$ mRNA was quantified by real-time rt-PCR in an Applied Biosystems 7500 Real-Time PCR System using genespecific Taq-Man Gene Expression Assays (Applied Biosystems Inc., Foster City, CA), and then normalized to $\beta$-actin mRNA. The following probes were used, all purchased from Applied Biosystems: G6Pase- $\beta$, Mm00616234_m1; G6PT, Mm00484574_m1; GRP78, Mm00517691_m1; GRP170, Mm00491278_g1, caspase-3, Mm00438045_m1 and $\beta$-actin, Mm00607939_s1.

\section{Western Blot Analysis of G6Pase- $\beta$}

Protein from mouse thigh-muscle was retrieved from the inorganic fraction of the TRIzol RNA isolation procedure (Invitrogen Corporation, Carlsbad, CA). Samples were resolved by electrophoresis through a $12 \%$ polyacrylamideSDS gel and trans-blotted onto polyvinylidene fluoride membranes (Millipore Co., Bedford, MA, USA). The membranes were incubated overnight with the polyclonal rabbit anti-Glc-6-Pase- $\beta$ antibody targeting amino acids 71-114 of the human G6Pase- $\beta$ (Chou Lab, NIH, UK) and then with horseradish peroxidase-conjugated goat anti-rabbit IgG (Kirkegarrd \& Perry Laboratories, Gaithersburg, MD, USA). The immunocomplex was visualized using the SuperSignal West Pico Chemiluminescent substrate from Pierce (Thermo Scientific, Rockford, IL).

\section{RESULTS}

Mice that are heterozygous or homozygous for the G6Pase- $\alpha$ gene are generally indistinguishable at all stages in life. However, mice that are homozygous null for G6Pase- $\alpha$ present with low birth weight, quickly develop severe and unremitting hypoglycemia, and gradually display increasing hyperlipidemia and osteodysmorphism. These problems worsened as the animals approached weaning. Their relative body mass also fell from 28 to $45 \%$ undersize. Without additional care at this point, weanlings inevitably died of hypoglycemia. These observations are in agreement with previously published findings. ${ }^{1,5,16-18}$

\section{Knockout Mice Undergo Intrinsic Physiological Improvement Post Weaning}

Mice treated palliatively with glucose injections, glucosefortified water and food supplementation had a $60 \%$ survival rate through weaning. Beyond this point, mice underwent significant transition, normalizing to various degrees in many disease-related parameters. Although body weight of $\mathrm{KO}$ pups averaged one-third below normal, $\mathrm{KO}$ adults finished growing just $5 \%$ beneath the median mass for healthy siblings. $\mathrm{KO}$ mice also showed marked improvement in mobility and activity. Liver-to-body weight improved, but remained above normal range, and kidney-to-body weight did not change with relation to normal animals (Figure 1). Although $\mathrm{KO}$ pups averaged $677 \mathrm{mg}$ per $100 \mathrm{ml}$ total cholesterol and $1720 \mathrm{mg}$ per $100 \mathrm{ml}$ triglycerides before weaning, these numbers fell to a mean of just $215 \mathrm{mg}$ per $100 \mathrm{ml}$ total cholesterol and $205 \mathrm{mg}$ per $100 \mathrm{ml}$ triglycerides by maturity. The average serum lipid levels from wild-type (WT) mice were 144 and $161 \mathrm{mg}$ per $100 \mathrm{ml}$, and more than half of adult KO mice were within a normal range. Circulating blood UA levels did not differ substantially between normal and KO animals of any age (Figure 2c).

Blood glucose measurements transitioned rapidly near weaning, from pups with severe and unremitting hypoglycemia to adults with general normoglycemia (70-200 mg per $100 \mathrm{ml}$ ) (Figure 2d) with only intermittent hypoglycemic events. Mice that became mildly hypoglycemic were usually capable of rebound, and $50 \mathrm{mg}$ per $100 \mathrm{ml}$ blood glucose was found to represent a general cut-off for normal activity, behavior and feeding. Although transient, survivable episodes of more severe hypoglycemia were intermittent throughout the 6-month time course, the longest-lived $\mathrm{KO}$ mice were able to maintain serum glucose levels above $50 \mathrm{mg}$ per $100 \mathrm{ml}$ on $92 \%$ of the days measured (Figure $2 \mathrm{~d}$ ).

\section{Liver and Kidney Histology does not Appear to Improve with Age}

Histological analysis of the liver tissue revealed distortion of the hepatic microarchitecture in both young and mature $\mathrm{KO}$ mice (Figure 3a and c) compared with that in WT animals (Figure $3 b$ and $d$ ). PAS-stained sections revealed excess glycogen accumulation in GSDIa mice compared with that in WT mice, independent of age (Figure 3a,c vs b,d). Analysis of histology and glycogen content revealed the same phenotypic manifestation in the kidney of $\mathrm{KO}$ animals compared with that in the kidney of WT animals (Figure 3e,g $v s \mathrm{f}, \mathrm{h}$ ). Aperio slide scanning and image analysis software were used to quantify relative staining intensity among mice of both genotypes and age brackets. Data revealed a clear genotype-dependent trend in glycogen storage, with mean staining intensity lowest in G6Pase $+/+$ mice, slightly higher in G6Pase $+/$ - mice and highest in $\mathrm{KO}$ mice. 


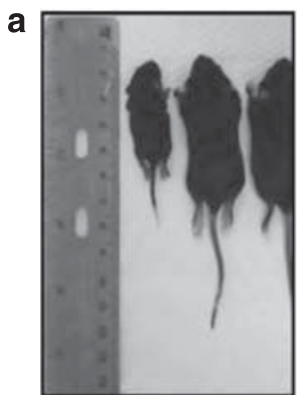

2 wks

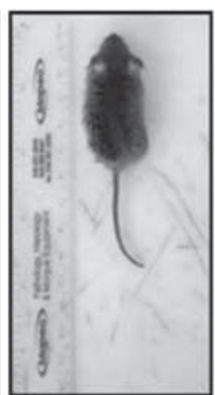

$1 \mathrm{mo}$

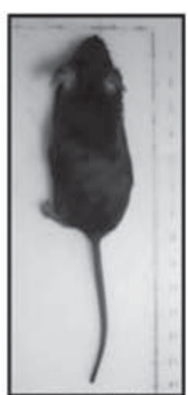

2 mo

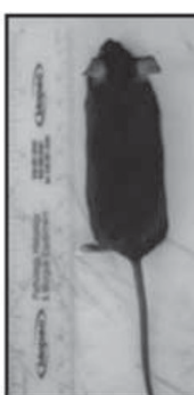

$3 \mathrm{mo}$

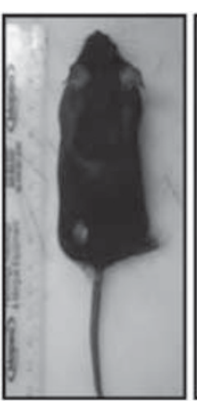

4 mo

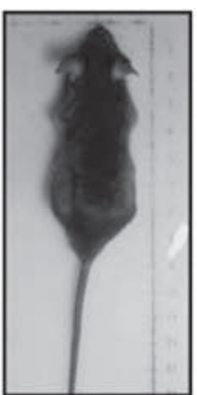

$5 \mathrm{mo}$

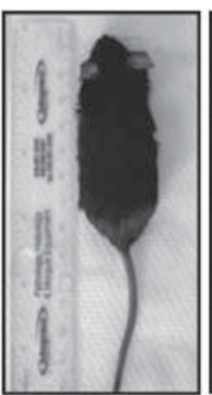

$6 \mathrm{mo}$

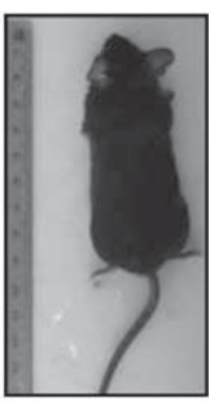

WT

b

body weight

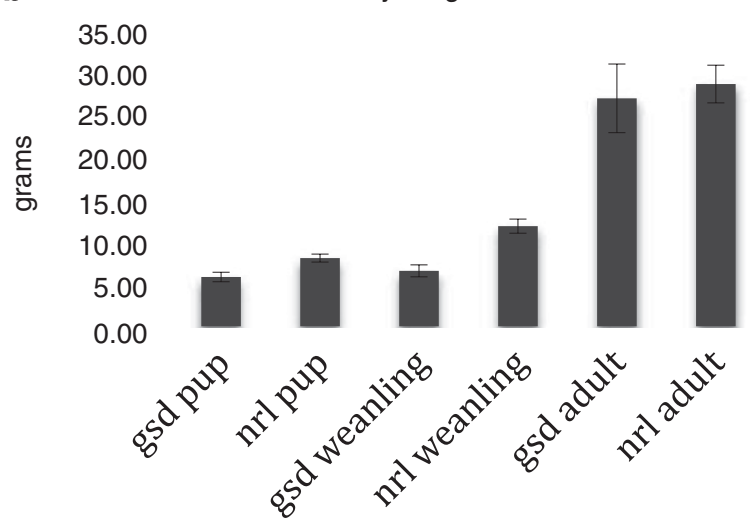

C

$15.00 \%$

liver:body weight

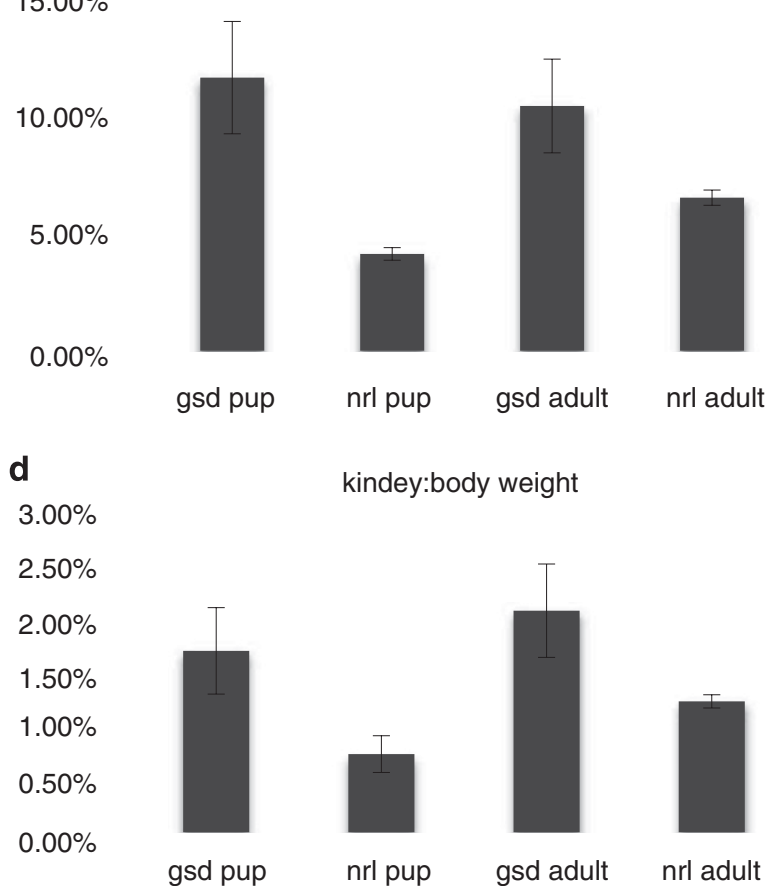

Figure 1 G6Pase-/- mice undergo innate morphological change as animals reach maturity. Pups that were markedly undersized were often determined to be homozygous null for G6Pase. (a) Pictures of representative KO mice with ages spanning from 2 weeks to 4 months with age-matched WT siblings at each end. Mice are pictured at the same scale. (b) Total body weight of mice from six representative categories. Weights represent pups at 11 days old

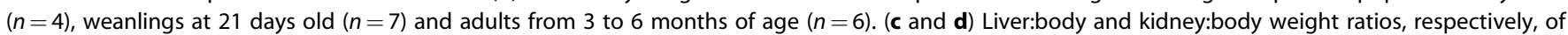
pups and adults (' $n$ ' as above). mo, months; wks, weeks. 


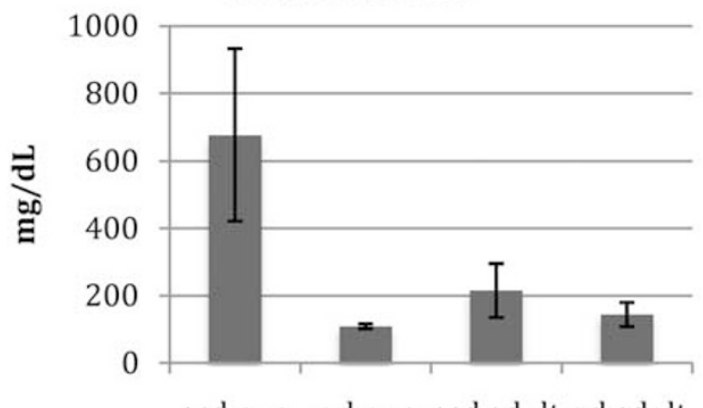

gsd pup nrl pup gsd adult nrl adult

b

Triglyceride

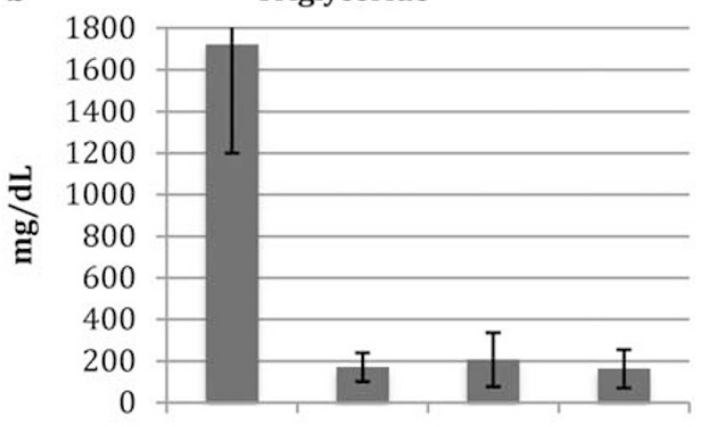

gsd pup nrl pup gsd adult nrl adult

C

Uric Acid

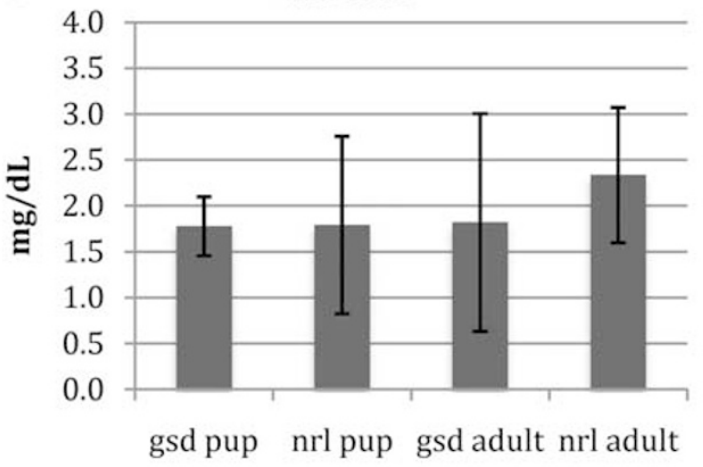

d

Daily Blood Glucose Monitoring

- L7.17.078 - L7.16.07 mLE

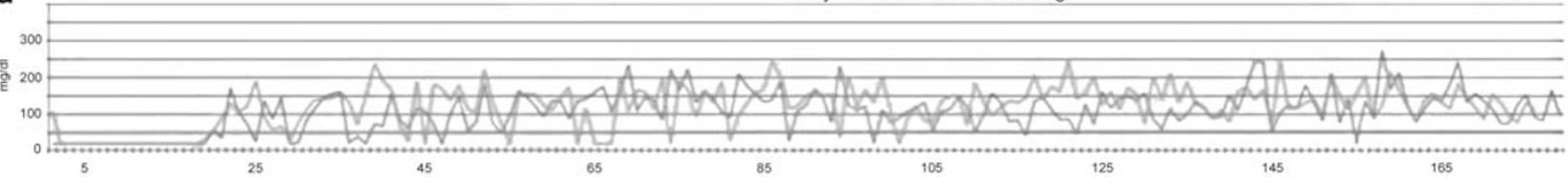

Figure 2 G6Pase-/- mice undergo innate change in serum chemistry as animals reach maturity. (a-c) Serum panels from pups aged 11 days $(n=4)$ and adults aged 3-6 months $(n=6)$ charting total cholesterol, triglycerides and uric acid, respectively. (d) Blood glucose readings from the oldest KO mice, studied over 6 months $(n=2)$. Mice were monitored for a total of 177 days, 155 of which were after the normal weaning point.

An age-dependent trend was also apparent, with younger mice storing slightly more glycogen than the older mice of their same genotype. ANOVA statistical analysis of staining intensity across the six groups of animals produced a $P$-value of 0.078 with $95 \%$ confidence level. To ensure that the magenta hue resulting from the PAS stain was indeed staining intracellular glycogen, another set of representative KO liver sections were stained with either PAS plus diastase digestion or PAS-only. Diastase digestion removed the majority of the magenta staining from PAS-treated liver sections, confirming that the staining was representative of glycogen within the fixed liver tissue (Supplementary Figure 1). Trichrome 
a

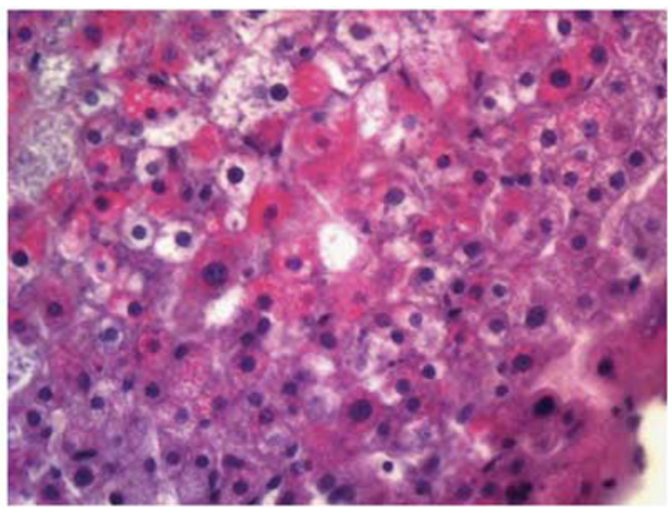

c

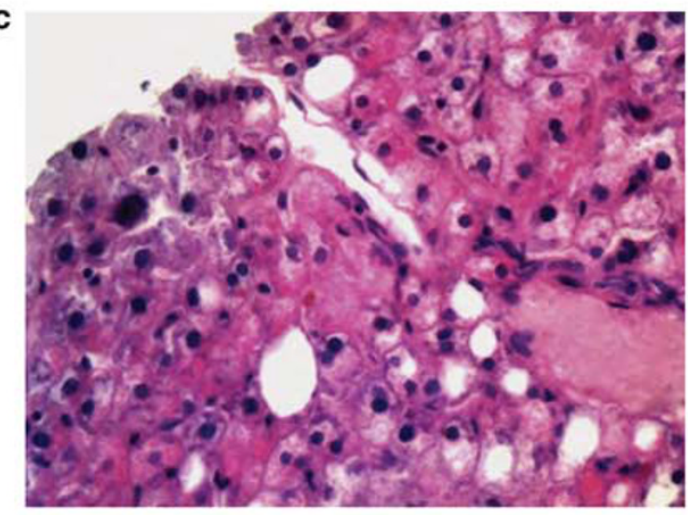

e

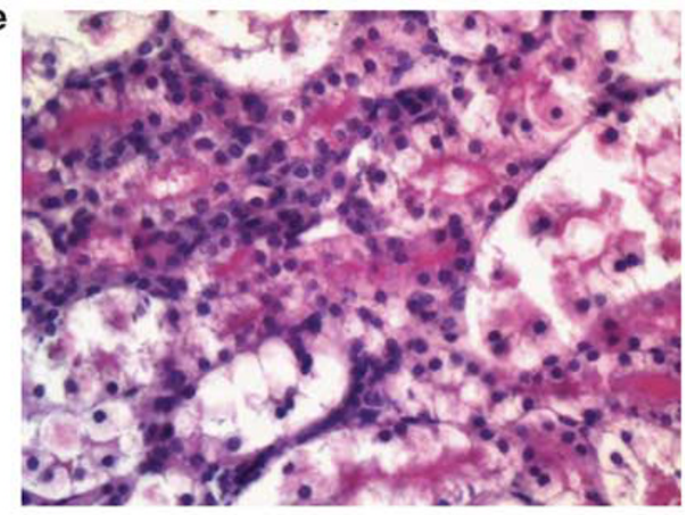

g 6

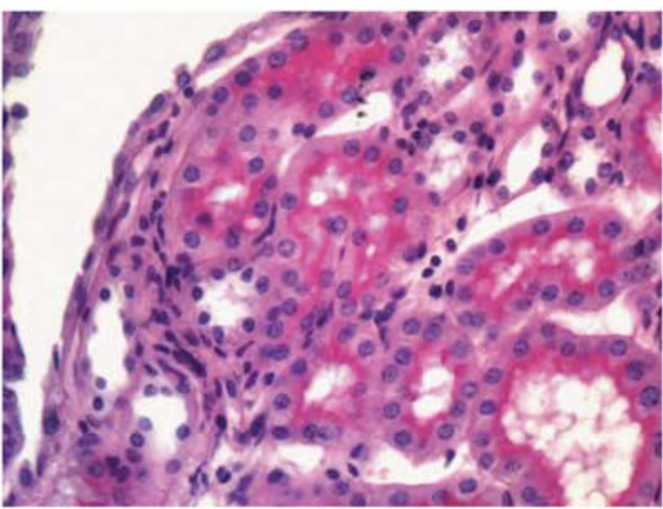

b

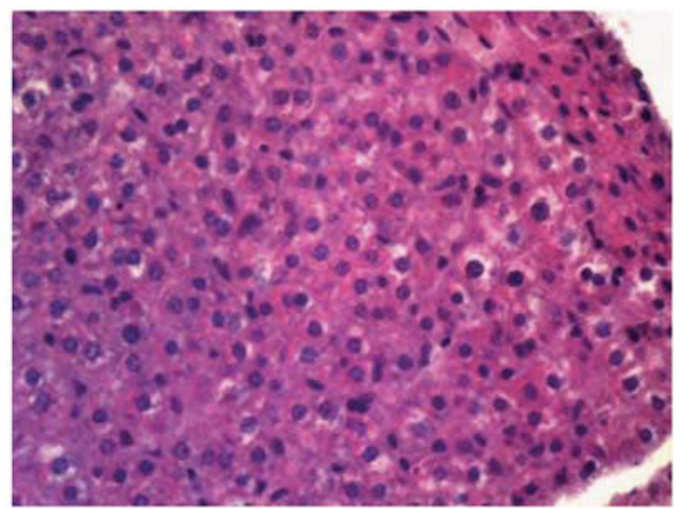

d

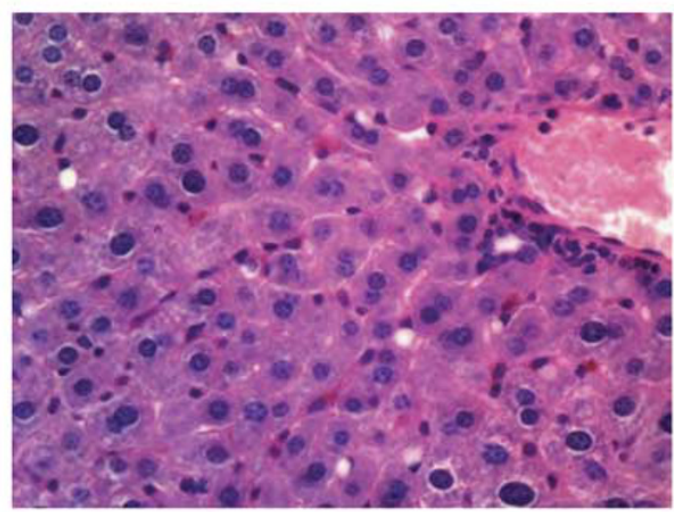

f

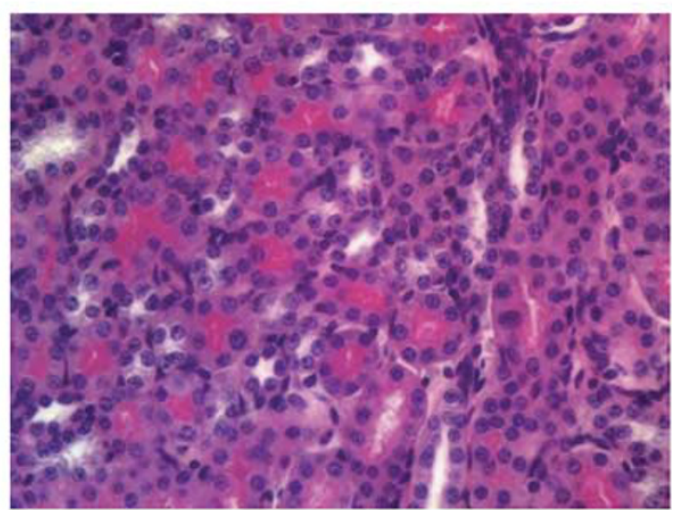

h

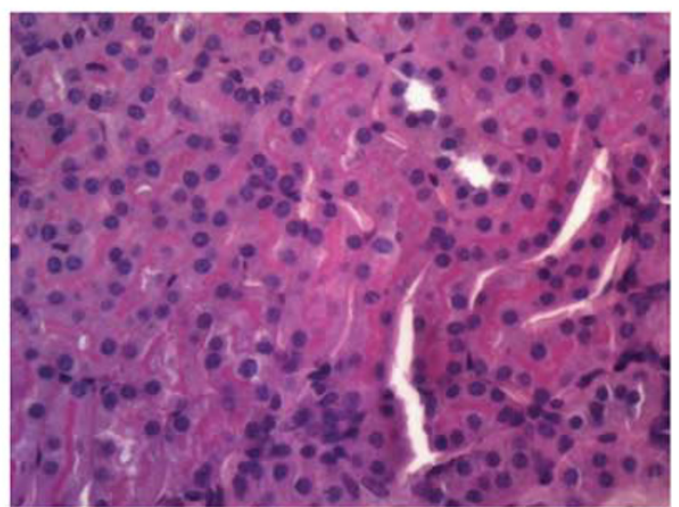

Figure 3 Histology and glycogen storage in the liver and kidneys of KO mice does not improve markedly with age. PAS-stained liver (a-d), and kidney (eh), from KO pup, WT pup and KO adult. Relative glycogen deposition is indicated by accumulation of magenta staining within the cell cytoplasm. All shown at $\times 400$ total magnification. 
a s

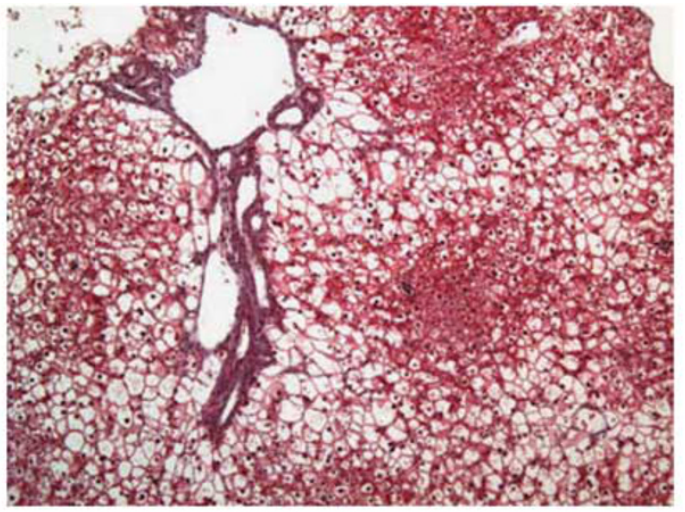

C

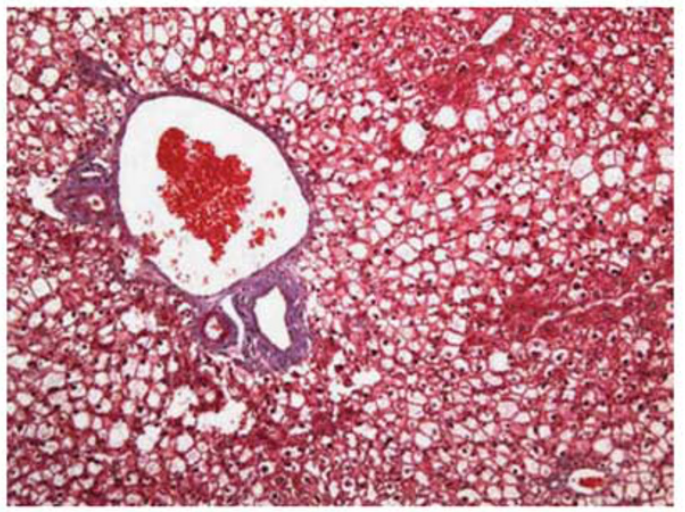

e

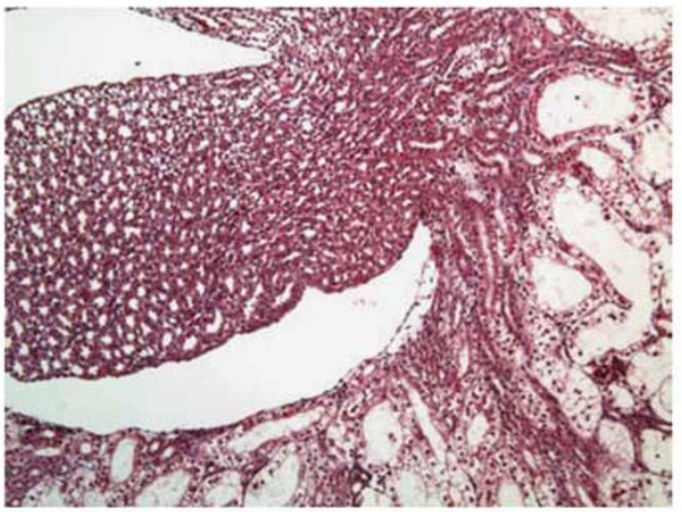

g

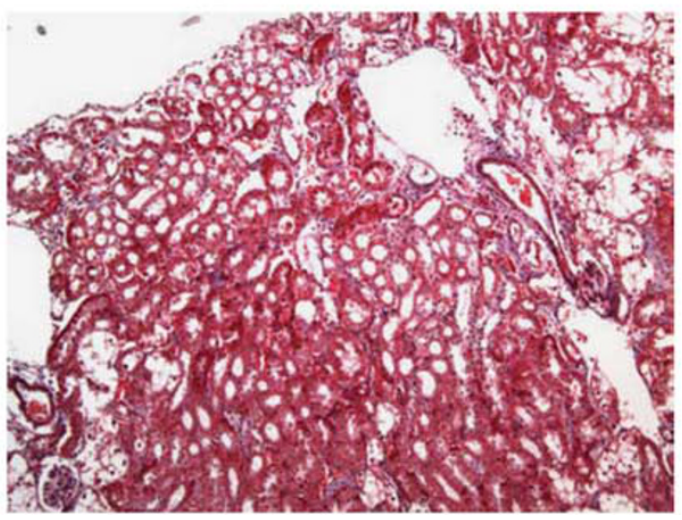

b

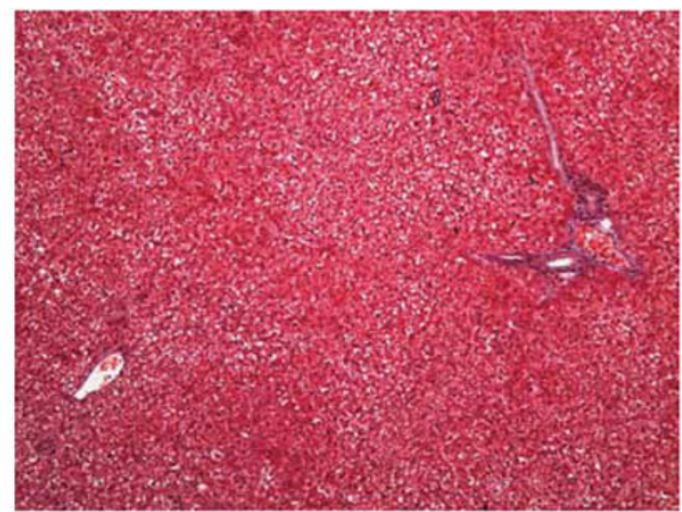

d

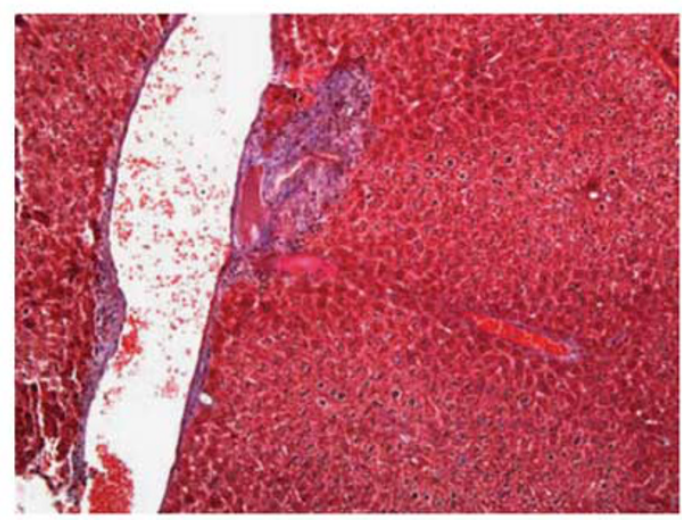

f

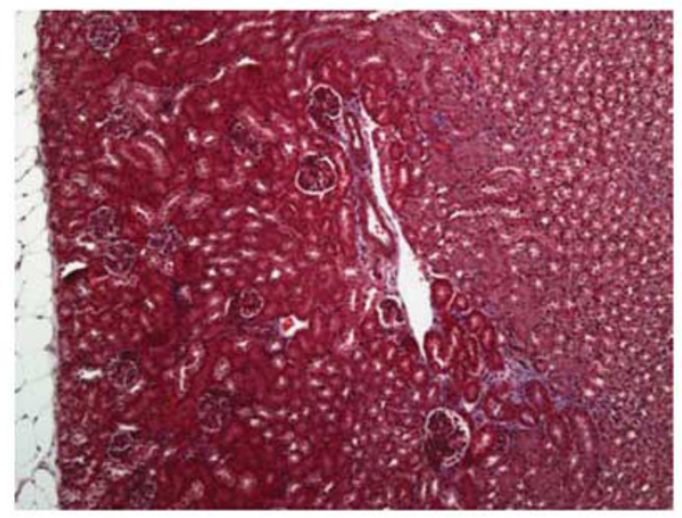

h

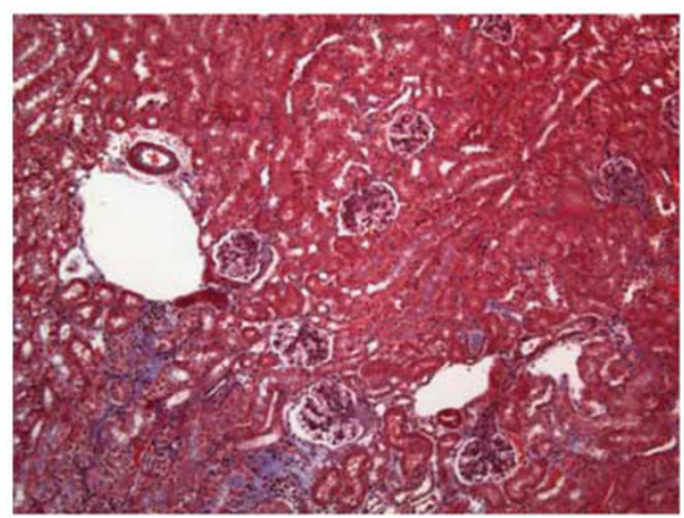

Figure 4 Fibrosis in the liver and kidney of KO mice does not worsen markedly with age. Masson's Trichrome-stained liver (a-d) and kidney (e-h) from KO pup, WT pup, KO adult and WT adult. Relative fibrosis is indicated by blue-stained tissue collagen. All shown at $\times 100$ total magnification. 
staining for type 1 collagen did not reveal significant fibrosis of either the liver or kidneys of $\mathrm{KO}$ animals (Figure 4).

\section{GSDla Adult Mice can Breed Naturally and Produce Viable Litters}

Successful mating of G6Pase-/- males to heterozygous females produced the expected 1:1 ratio of heterozygous and null pups (Figure 5a). Surprisingly, female KO mice were capable of withstanding pregnancy (Figure $5 b$ ), and a cross between two KO parents produced normal sized litters of $100 \%$ KO pups. The G6Pase- $\alpha-/-$ female maintained safe blood sugar levels throughout the majority of the pregnancy, and had uncomplicated delivery. Approximately $90 \%$ of the pups were viable at birth; however, the mother was unable to nurse adequately. Providing a healthy lactating surrogate female will negate this problem, should G6Pase-/- mothers be unable to nurse future litters.

\section{G6Pase- $\beta$ is not Upregulated with Age in GSDla Mice}

G6Pase- $\beta$ regulation was evaluated at the level of transcription, translation and functional phosphohydrolase activity. Quantitative real-time rt-PCR showed no significant upregulation of G6Pase- $\beta$ transcription in the muscle of GSDIa mice as compared with that in WT animals (Figure 6a). In agreement with this, western blot analysis of protein extracts from muscle tissue did not reveal differential regulation of the $\beta$ isoform between G6Pase- $\alpha$ and wild-type mice (Figure $6 \mathrm{~b})$. The phosphatase activity assay confirmed the absence of G6Pase activity in the liver of GSDIa mice at each age tested (data not shown). Although recent investigations employed
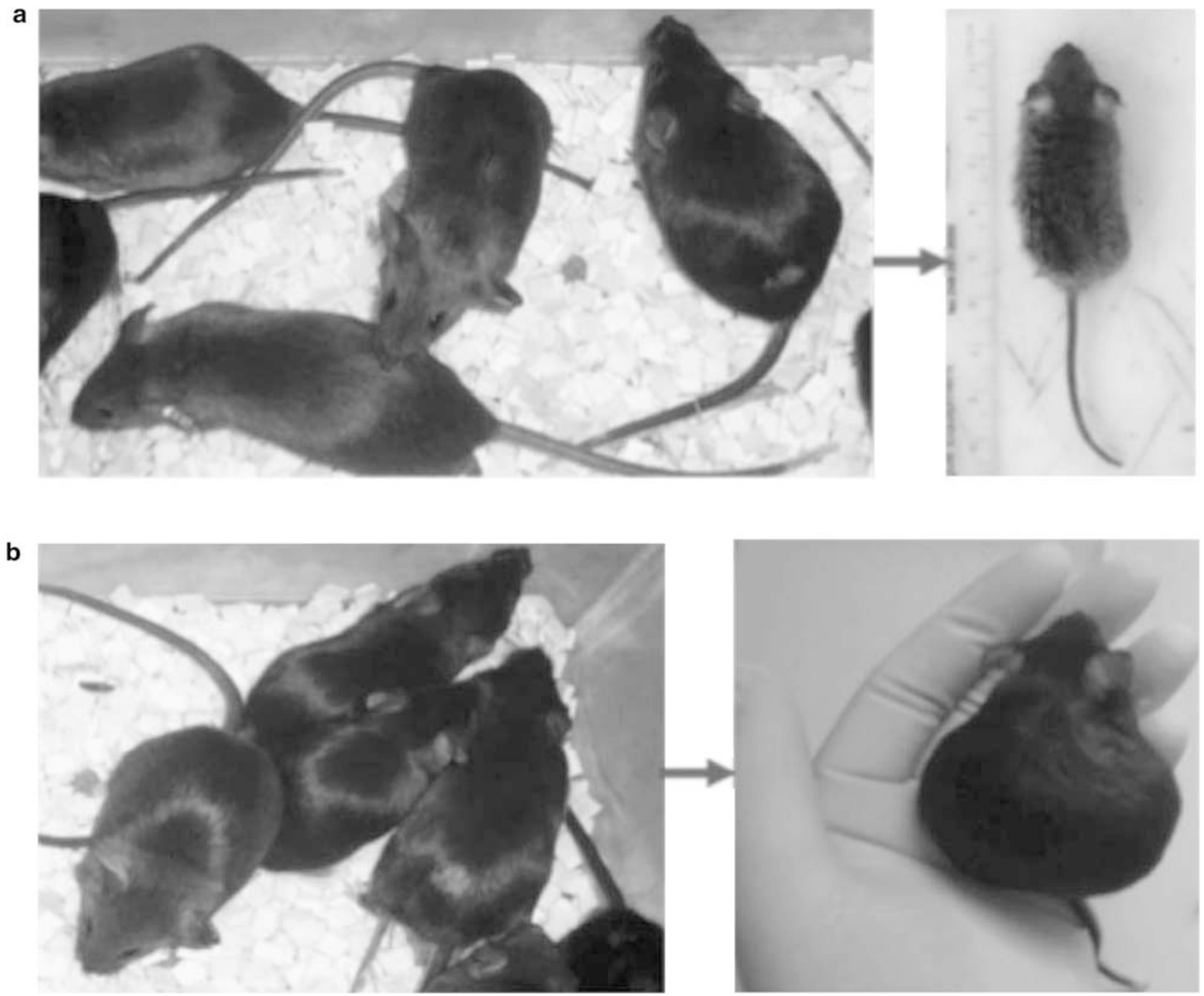

Figure 5 G6Pase-/- mice of both genders are able to breed to produce viable litters. (a) Male KO mouse (black, top right) $\times$ female heterozygote (brown, bottom left) produced first reported litter from a KO parent. Representative KO progeny shown at right. (b) Male KO (black, right) $\times$ female KO (black, center) produced first reported $100 \%$ KO litter. Pregnant KO female shown at right. 
a

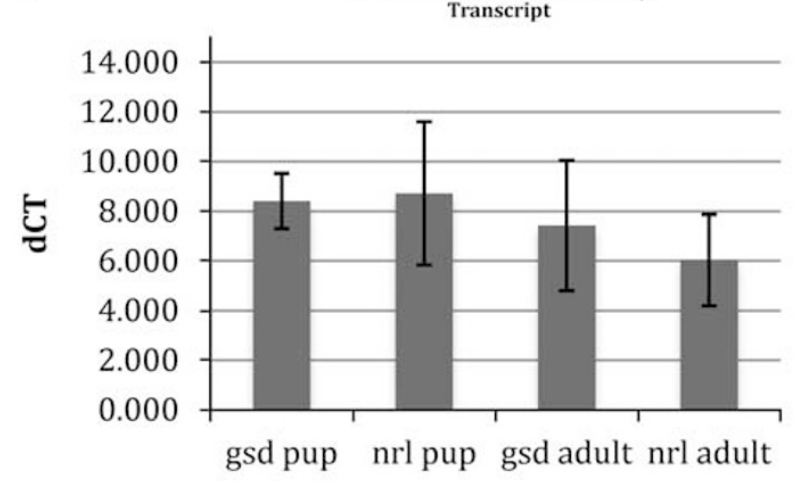

b G6Pase- $\beta$

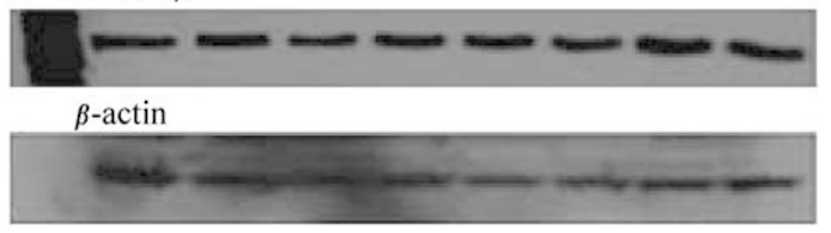

Figure 6 G6Pase- $\beta$ was not differentially expressed in KO (G6Pase- $\alpha-/-$ ) animals relative to WT. (a) Quantitative rt-PCR of G6Pase- $\beta$ mRNA in muscle tissue from adults and pups of both genotypes (KO $n=5$ and WT $n=4$, both age groups). Numbers represent difference in target copy number relative to $\beta$-actin; therefore, a lower mean value indicates higher expression (b) Western blot of G6Pase- $\beta$ protein in muscle tissue from adult KO (right) and WT (left) mice ( $n=4$, both). Membranes were stripped and re-probed for $\beta$-actin (shown beneath G6Pase- $\beta$ blot).

an in vitro overexpression system to examine G6Pase- $\beta$ kinetics, ${ }^{12,15}$ this approach was not available for our study. These results suggest that G6Pase- $\beta$ upregulation is not an adaptive mechanism in post-weaning G6Pase $\mathrm{KO}$ mice.

\section{DISCUSSION}

As GSDIa was first described in 1929, nutritional protocols for both children and adults with the disorder have been carefully defined for optimal maintenance of health. The meticulous dietary guidelines have been made available in many formats, and with strict adherence and interprandial supplementation, patients with GSDIa have a fairly good prognosis. Even so, the lifestyle required to maintain the health of a child with GSDIa is an incredible challenge. The severity of symptoms is often more pronounced in childhood, and the potential for the next life-threatening hypoglycemic event is ever-present. Careful management and continued dietary adherence is a continuing battle, but meticulous glycemic control can produce striking clinical and metabolic improvement, and is closely linked with overall prognosis. ${ }^{19,20}$ Moreover, the severity of symptoms is known to lessen with age $\left(2002 \mathrm{chou} / \mathrm{chen},{ }^{16} 2003 \mathrm{Shieh} / \mathrm{Chou},{ }^{12}\right.$ 2004 Shieh/Chou $\left.{ }^{15}\right)$. Nonetheless, the threat of acute hypoglycemia, malnutrition, osteoporosis, hyperlipidemia, hepatic malignancy, and the liver or kidney disease never subsides throughout the patient's lifetime. To date, orthotropic liver transplantation is the only means available to correct the genetic disorder. However, beyond the risks implicit to allogenic transplantation, GSDIa patients also have a heightened chance for complications in long-term management. $^{2}$ Therefore, an alternative means for eradicating the disease is essential for offering a chance at a normal life.

Over the past 10 years or so, a number of translational studies have set out to eliminate the manifestations of GSDIa utilizing a gene therapy approach to shuttle exogenous G6Pase- $\alpha$ to KO animals. ${ }^{1,5,16-18,21}$ Throughout the cited literature, untreated G6Pase KO pups were shown to suffer acutely from classical disease-related symptoms including stunted growth, unremitting hypoglycemia, and pronounced elevation in serum cholesterol, triglyceride and UA levels. Survival of afflicted pups beyond weaning has been described as a 'rare occurrence' unless some type of intervention was implemented. To the contrary, these studies clearly showed that KO pups treated with adeno- or AAV viral vectors at 2 weeks of age not only survived weaning, but also showed phenotypic improvement by week 4 , and were virtually indistinguishable from WT littermates with respect to each disease parameter measured by maturity. ${ }^{21}$

The high fatality of KO pups had eliminated the chance for comparison between vector-treated and untreated adults. Thus, earlier studies relied on the phenotype of immature KO pups as a baseline for gauging the long-term effects of gene therapy. In this setting, vector administration was concluded to have caused the improved health of treated animals. ${ }^{21}$ Notably, however, untreated KO pups that survived to the weaning in these studies also showed moderate increase in average blood glucose, with values falling between those of afflicted neonates and normal age-matched siblings. Most remarkably, the few untreated $\mathrm{KO}$ mice that reported to survive post weaning displayed marked improvements in serum and growth profiles as well. ${ }^{21}$ Although these data were noted graphically, its significance has not been addressed.

To our knowledge, no earlier study had considered the potential for natural adaptation of $\mathrm{KO}$ mice in the evaluation of GSDIa treatment efficacy. In the absence of gene therapy, we observed remarkable improvement in serum lipid profiles, glycemic control, size, appearance and liveliness. We also observed some relative improvement in hepatomegaly, but little change in nephromegaly, or liver lobule microarchitecture. Not surprisingly, PAS staining of both tissues revealed that glycogen accumulation did not improve with age; however, Masson's Trichrome stain for type 1 collagen did not reveal development of pathological fibrosis. Interestingly, although lethal hepatic and renal lesions have been reported in untreated KO pups by $4-5$ weeks of age, ${ }^{16}$ this result was not reproduced in our study, even in mice as old as 6 months. Though the reason for this discrepancy is unclear, dietary supplementation with Nutragel likely contributed to the relative health of our animals. 
In the clinic, G6Pase deficiency leaves patients highly susceptible to hyperuricemia from the overproduction and under-excretion of urate. In humans, build up of lactic acid from alternative metabolic breakdown of G6P only worsens the hyperuricemia, as UA must compete with lactic acid for excretion by a common renal anion transporter. ${ }^{22}$ For yet unknown reasons, the $\mathrm{KO}$ mice do not typically manifest lacticacidemia. With normal lactic acid levels, KO mice would have a greater capacity to eliminate circulating UA and avoid hyperuricemia, as observed in our study of the model.

The robustness of the adult G6Pase $\mathrm{KO}$ mouse was again shown when KO males produced viable litters with both heterozygous and $\mathrm{KO}$ females. Notably, female $\mathrm{KO}$ mice were able to carry litters of normal size to term, and deliver without complication. In common with some human patient reports (personal communication with Dr David Weinstein), KO mice did not successfully nurse, but providing a healthy female surrogate may allow for rearing of $100 \% \mathrm{KO}$ litters. The capacity for such high-throughput production is particularly useful to this model, as a fraction of $\mathrm{KO}$ pups in each litter will die despite the best available care. Also alternative to the standard heterozygote cross, breeding heterozygous females with KO males will still double the efficiency of producing $\mathrm{KO}$ pups in numbers suitable for studies of age-matched littermates, without the need for a surrogate mother.

Upregulation of a G6Pase isoform, G6Pase- $\beta$, has been postulated as a partial explanation behind the phenotypic improvement in humans. With the animal model capable of an even more drastic change, it was important to investigate whether the transition was due to upregulation of a compensatory enzyme, in addition to modifications in husbandry. As the largest glycogen reserves are found not in the liver, but throughout the skeletal muscle; it would then be logical to suspect that some analog of G6Pase might also exist in association with this tissue. The initial published characterization explored the enzyme's gluconeogenic potential first in vitro, and then in vivo using the young GSDIa mouse to eliminate background from G6Pase- $\alpha$ activity. ${ }^{12,15,23}$ The results showed that G6Pase- $\beta$ phosphohydrolase has $12 \%$ of the activity of hepatic G6Pase- $\alpha$. The in vivo study also revealed that G6Pase- $\beta$ transcript, protein and enzyme activity levels were roughly equivalent among young WT and G6Pase- $\alpha-1-$ mice, ${ }^{15}$ thus, providing evidence that G6Pase- $\beta$ is not differentially enhanced in young mice lacking G6Pase- $\alpha$. With the KO mouse now available for analysis throughout the stages of development, our study addressed the possibility that upregulation of this secondary enzyme could be responsible for age-dependent improvement.

Characterization of our G6Pase- $\alpha-/-$ mice verified that, per unit mass of tissue, G6Pase- $\beta$ is not upregulated with age (see Figure 6). Quantification of G6Pase- $\beta$ by real-time rt-PCR and western blot analysis did not show an increase in G6Pase- $\beta$ transcript and protein expression, respectively, and the activity assay did not suggest enhancement of muscle-bound G6Pase- $\beta$ to nearly the magnitude of the functional hepatic isoform (data not shown). Furthermore, as no other related enzymes were predicted by genomic search, ${ }^{12}$ it is unlikely that a third yet-unknown analog is responsible for the improvement. Despite the absence of upregulation, it is still probable that G6Pase- $\beta$ can play a role in age-related improvement. As relative muscle-to-body mass increases from $20 \%$ in neonates to $40-45 \%$ in adults, ${ }^{15}$ a greater percentage of tissue is able to produce the gluconeogenic enzyme. Although the clinical importance of muscle-bound G6Pase- $\beta$ will have to be explored with human tissue, increased muscle mass very likely contributes to the improved metabolic control that patients experience as they mature. Moreover, the patient's increased response to physiological cues and steady dietary optimization is also likely to have a role in the lessening of their symptoms.

Mice undergo a more pronounced improvement in robustness than humans do because mouse pups manifest the disease much more severely. The reasons for this discrepancy are not exhaustively defined; however, the simple increase of bioavailable glucose that occurs with weaning is almost certainly behind the improvement seen in mice. Although human infants with GSDIa are fed a lactose-free formula, ${ }^{20}$ mouse pups continue to nurse exclusively. In the absence of G6Pase- $\alpha$, KO pups are unable to metabolize lactose efficiently, and much of the consumed nutritive value is inaccessible. Before weaning, malnutrition impedes development, and excess sugar is shunted down alternative metabolic pathways, precipitating hyperlipidemia. Replacement of milk with standard adult chow and easily ingestible high-calorie Nutragel supplement gives young mice a better balance of more accessible nutrition, at which point their condition improves dramatically. Additionally, the addition of glucose to their water allowed for a quick glycemic boost interprandially and led to improved survival rates.

Undoubtedly, the previous reports that employed gene therapy for the treatment of GSDIa have produced marked improvements in histology and glycogen accumulation with restoration of G6Pase- $\alpha$ activity. These changes alone verify the value of gene or cell-based therapies. It is also evident that the viral vectors have assisted afflicted pups through the preweaning 'crisis' period, where without any palliative support these animals would likely have died. However, our study reveals that relative glucose homeostasis, normalized serum panels, normal growth and even reproductive ability can be achieved by modifications in husbandry.

Our method for rearing G6Pase- $\alpha$ KO mice into maturity provides a critical tool, which may potentiate many advances within this research field. The adult KO mouse will provide a vital prerequisite for any investigational therapies requiring a larger and healthier pool of animals; particularly, for those procedures that employ cell transplantation and for those that wish to investigate gene therapy potential in fully matured animals. With our current knowledge of hepatic cell transplantation, certain procedures must be implemented to 
prime the hepatic niche for engraftment of transplanted cells. With rare exception, this priming requires chemical inhibition of endogenous cells, followed by physical injury to the liver. ${ }^{24,25}$ Sickly KO pups would not likely survive the preconditioning for engraftment, nor withstand the recovery period from surgical cell infusion. With the resilient adult mouse now available, research on hepatocyte and stem cell transplantation will now be much more feasible.

In summary, this detailed characterization of the mature KO mouse provides a critical standard for evaluating the true efficacy of potential treatments. The capacity to raise untreated GSD mice to maturity also provides the opportunity to test new treatment options; whereas direct stem cell transplantation to the GSD liver would be devastating in neonates and it would be relatively uncomplicated in mature animals. By breeding $\mathrm{KO}$ mice, we have also greatly improved our capacity to produce litters with KO pups in sufficient numbers for study. Finally, our work has shed new light on the parallel in age-related improvements seen in both mice and humans, and furthered our general understanding of this serious disease.

Supplementary Information accompanies the paper on the Laboratory Investigation website (http://www.laboratoryinvestigation.org)

\section{ACKNOWLEDGEMENTS}

The authors give theirs appreciation to $\mathrm{Dr}$ Janice Chou of the $\mathrm{NIH}$ for providing an introduction to the animal model, the research mice and antibody. We also thank the Children's Fund for Glycogen Storage Disease, and the Matthew Ehrman Fund for GSD Research for financially supporting this work.

1. Ghosh A, Allamarvdasht M, Pan C, et al Long-term correction of murine glycogen storage disease type la by recombinant adeno-associated virus-1-mediated gene transfer. Gene Ther 2006;13:321-329.

2. Davis $M$, Weinstein $D$. Liver transplantation in children with glycogen storage disease: Controversies and evaluation of the risk/benefit of this procedure. Pediatr Transplant 2008;12:137-145.

3. Lei KJ, Shelly LL, Pan CJ, et al Mutations in the glucose-6-phosphatase gene that cause glycogen storage disease type 1a. Science 1993;262:580-583.

4. Pan $\mathrm{CJ}$, Lei $\mathrm{KJ}$, Chen $\mathrm{H}$, et al Ontogeny of the murine glucose-6phosphatase system. Arch Biochem Biophys 1998;358:17-24.

5. Koeberl D, Sun B, Damodaran T, et al Early, sustained efficacy of adenoassociated virus vector-mediated gene therapy in glycogen storage disease type la. Gene Ther 2006;13:1281-1289.

6. Rosh JR, Collins J, Groisman GM, et al Management of hepatic adenoma in glycogen storage disease la. J Pediatr Gastroenterol Nutr 1995;20:225-228.
7. Labrune $\mathrm{P}$, Trioche $\mathrm{P}$, Duvaltier I, et al Hepatocellular adenomas in glycogen storage disease type I and III: a series of 43 patients and review of the literature. J Pediatr Gastroenterol Nutr 1997;24: 276-279.

8. Debaere C, Op de Beeck B, De Maeseneer $M$, et al Magnetic resonance imaging findings of hepatic adenomas in von Gierke (type I) glycogen storage disease: case report. Can Assoc Radiol J 1999;50:161-164.

9. Shelly LL, Lei KJ, Pan CJ, et al Isolation of the gene for murine glucose6-phosphatase, the enzyme deficient in glycogen storage disease type 1A. J Biol Chem 1993;268:21482-21485.

10. Lei KJ, Shelly LL, Lin B, et al Mutations in the glucose-6-phosphatase gene are associated with glycogen storage disease types 1a and 1aSP but not 1b and 1c. J Clin Invest 1995;95:234-240.

11. Beaty $R$, Jackson $M$, Peterson D, et al Delivery of glucose-6phosphatase in a canine model for glycogen storage disease, type la, with adeno-associated virus (AAV) vectors. Gene Ther 2002;9:1015-1022.

12. Shieh J. A Glucose-6-phosphate hydrolase, widely expressed outside the liver, can explain age-dependent resolution of hypoglycemia in glycogen storage disease type la. J Biol Chem 2003;278:47098-47103.

13. Cheung Y, Kim S, Yiu W, et al Impaired neutrophil activity and increased susceptibility to bacterial infection in mice lacking glucose6-phosphatase- $\beta$. J Clin Invest 2007;117:784-793.

14. Harper AE. Glucose-6-phosphatase. In: Methods of Enzymatic Analysis Bergmeyer HU (ed). New York: Academic 1965, pp 788-792.

15. Shieh J. A potential new role for muscle in blood glucose homeostasis. J Biol Chem 2004;279:26215-26219.

16. Chou J, Zingone A, Pan C. Adenovirus-mediated gene therapy in a mouse model of glycogen storage disease type 1a. Eur J Pediatr 2002;161:S56-S61.

17. Sun MS, Pan C, Shieh JJ, et al Sustained hepatic and renal glucose-6phosphatase expression corrects glycogen storage disease type la in mice. Hum Mol Genet 2002;11:2155-2164.

18. Koeberl D, Pinto C, Sun B, et al AAV vector-mediated reversal of hypoglycemia in canine and murine glycogen storage disease type la. Mol Ther 2008;16:665-672.

19. Moses S. Historical highlights and unsolved problems in glycogen storage disease type 1. Eur J Pediatr 2002;161:S2-S9.

20. Wolfsdorf Jl, Weinstein D. Glycogen storage diseases. Rev Endocr Metab Disord 2003:4:95-102.

21. Zingone $\mathrm{A}$, Hiraiwa $\mathrm{H}, \mathrm{Pan} \mathrm{CJ}$, et al Correction of glycogen storage disease type 1a in a mouse model by gene therapy. J Biol Chem 2000;275:828-832.

22. Raghavan VA, Kline GA, Corenblum B. Glucose-6-phosphatase deficiency. 2007 Medscape http://emedicine.medscape.com/article/ 119184-overview.

23. Martin CC, Oeser JK, Svitek CA, et al Identification and characterization of a human CDNA and gene encoding a ubiquitously expressed glucose-6-phosphatase catalytic subunit-related protein. J Mol Endocrinol 2002;29:205-222.

24. Aurich I, Mueller $\mathrm{L}$, Aurich $\mathrm{H}$, et al Functional integration of hepatocytes derived from human mesenchymal stem cells into mouse livers. Gut 2007;56:405-415.

25. Ikeno $M$, Inagaki $H$, Nagata $K$, et al Generation of human artificial chromosomes expressing naturally controlled guanosine triphosphate cyclohydrolase I gene. Genes Cells 2002;7:1021-1032. 